\title{
On the Impact of a Small Initial Population Size in the IPOP Active CMA-ES with Mirrored Mutations on the Noiseless BBOB Testbed
}

\author{
Dimo Brockhoff \\ INRIA Lille - Nord Europe \\ Dolphin team \\ 59650 Villeneuve d'Ascq \\ France \\ dimo.brockhoff@inria.fr
}

\author{
Anne Auger \\ Projet TAO, INRIA \\ Saclay-lle-de-France \\ LRI, Bât 490, Univ. Paris-Sud \\ 91405 Orsay Cedex, France \\ anne.auger@inria.fr
}

\author{
Nikolaus Hansen \\ Projet TAO, INRIA \\ Saclay-lle-de-France \\ LRI, Bât 490, Univ. Paris-Sud \\ 91405 Orsay Cedex, France \\ nikolaus.hansen@inria.fr
}

\begin{abstract}
Active Covariance Matrix Adaptation and Mirrored Mutations have been independently proposed as improved variants of the well-known optimization algorithm Covariance Matrix Adaptation Evolution Strategy (CMA-ES) for numerical optimization. This paper investigates the impact of the algorithm's population size when both active covariance matrix adaptation and mirrored mutation are used in the CMA-ES. To this end, we compare the CMA-ES with standard population size $\lambda$, i.e., $\lambda=4+\lfloor 3 \log (D)\rfloor$ with a version with half this population size where $D$ is the problem dimension.
\end{abstract}

\section{Categories and Subject Descriptors}

G.1.6 [Numerical Analysis]: Optimization-global optimization, unconstrained optimization; F.2.1 [Analysis of Algorithms and Problem Complexity]: Numerical Algorithms and Problems

\section{General Terms}

Algorithms

\section{Keywords}

Benchmarking, Black-box optimization

\section{INTRODUCTION}

The IPOP-CMA-ES [2] has the special feature of increasing the population size of the CMA-ES algorithm at each restart. Together with a standard population size of $\lambda^{s}=$ $4+\lfloor 3 \log (D)\rfloor$ where $D$ is the problem dimension, the IPOPCMA-ES is a (nearly) parameterless algorithm that automatically restarts CMA-ES with increased population size if the given size is not sufficient to solve the problem at hand.

(C)ACM, 2012. This is the authors' version of the work. It is posted here by permission of ACM for your personal use. Not for redistribution. The definitive version was published at GECCO Companion '12, July 7-11, 2012, Philadelphia, PA, USA. http://doi.acm.org/10.1145/2330784.2330825
More recently, an active covariance matrix adaptation update has been proposed for CMA-ES [9] and mirrored mutations with pairwise selection and selective mirroring have been suggested for evolution strategies with weighted recombination [1]. While the former one allows for negative weights in the covariance matrix update for bad mutations, the latter mirrors the bad mutations and evaluates them again before to proceed.

The combination of both approaches into the IPOP-CMAES with active covariance matrix adaptation and mirrored mutations, denoted by $\mathrm{CMA}_{\mathrm{ma}}$, has been introduced and tested empirically in an accompanying paper [3]. Here, we test how a different starting population size influences the performance of this algorithm. A previous study showed that in the $(1, \lambda)$-ES, the largest effect of mirrored mutations is observed for small population sizes, i.e. $\lambda=2$ and $\lambda=4$ [4]. Hence, we could conjecture that in the IPOPCMA-ES with mirrored mutations, a positive effect on the performance can be observed if the initial population size is chosen smaller than the standard size of $\lambda^{s}$. To test this hypothesis, we run the $\mathrm{CMA}_{\text {ma }}$ with an initial population size of $\lambda^{s}$ and compare it with the $\mathrm{CMA}_{\text {mah }}$ that employs an initial population size of $\left\lfloor\lambda^{s} / 2\right\rfloor$ on the noiseless BBOB test bed [6].

The algorithms are described in more detail in Sec. 2. Section 3 gives the mandatory results of the BBOB timing experiments while Sec. 4 presents the general results of the comparison. Section 5 concludes the paper.

\section{TESTED CMA-ES VARIANTS}

We tested two variants of the IPOP-CMA-ES with active covariance matrix adaptation and mirrored mutations: the $\mathrm{CMA}_{\text {ma }}$ with standard initial population size $\lambda^{s}=4+$ $\lfloor 3 \log (D)\rfloor$ and the $\mathrm{CMA}_{\text {mah }}$ with reduced initial population size $\left\lfloor\lambda^{s} / 2\right\rfloor$. Both implementations can be downloaded from http://canadafrance.gforge.inria.fr/mirroring/ in the used version 3.54.beta.mirrors. Besides the difference in the initial population size, the number of restarts is increased to 10 for the $\mathrm{CMA}_{\text {mah }}$ instead of 9 for the $\mathrm{CMA}_{\text {ma }}$ to allow the final restarts of both algorithms to operate with the same (range of) population size. All other parameters are equal for the two algorithms and, besides $2 \cdot 10^{5} \cdot D$ as the maximal number of function evaluations, chosen according to the standard recommendations for the CMA-ES. For 
more details of the algorithm, see also the accompanying paper [3].

\section{TIMING EXPERIMENTS}

In order to see the dependency of the algorithms on the problem dimension, the requested BBOB'2012 timing experiment has been performed for the two algorithms $\mathrm{CMA}_{\mathrm{ma}}$ and $\mathrm{CMA}_{\text {mah }}$ on an Intel Core2 Duo T9600 laptop with $2.80 \mathrm{GHz}, 4.0 \mathrm{~GB}$ of RAM, and MATLAB R2008b on Windows Vista SP2. The algorithms have been restarted for up to $2 \cdot 10^{5} \mathrm{D}$ function evaluations until 30 seconds have passed. The per-function-evaluation-runtimes were $16 ; 16 ; 11 ; 6.5$; 4.2 ; 4.6 ; and 7.2 times $10^{-4}$ seconds for the $\mathrm{CMA}_{\mathrm{ma}}$ and $21 ; 19 ; 11 ; 8.3 ; 6.1 ; 5.7$ and 11 times $10^{-4}$ seconds for the $\mathrm{CMA}_{\text {mah }}$ in $2,3,5,10,20,40$, and 80 dimensions respectively.

\section{RESULTS}

Results from experiments according to [6] on the benchmark functions given in $[5,7]$ are presented in Figures 1, 2 and 3 and in Tables 1 . The expected running time (ERT), used in the figures and table, depends on a given target function value, $f_{\mathrm{t}}=f_{\mathrm{opt}}+\Delta f$, and is computed over all relevant trials as the number of function evaluations executed during each trial while the best function value did not reach $f_{\mathrm{t}}$, summed over all trials and divided by the number of trials that actually reached $f_{\mathrm{t}}[6,10]$. Statistical significance is tested with the rank-sum test for a given target $\Delta f_{\mathrm{t}}$ $\left(10^{-8}\right.$ as in Figure 1) using, for each trial, either the number of needed function evaluations to reach $\Delta f_{\mathrm{t}}$ (inverted and multiplied by -1 ), or, if the target was not reached, the best $\Delta f$-value achieved, measured only up to the smallest number of overall function evaluations for any unsuccessful trial under consideration.

The first observation is the fact that both algorithm variants behave quite similar with only a few cases where the differences are statistically significant. The two main exceptions are the sphere function $\left(f_{1}\right)$ for which the variant with smaller initial population size is about $25 \%$ faster in all dimensions and all difficult targets and the discus function $\left(f_{11}\right)$ where the variant with standard population size is about $20 \%$ faster in $5 \mathrm{D}$ and $10 \%$ faster in $20 \mathrm{D}$ (see Table 1). Figure 1 reveals a few more statistically significant differences for the target value $10^{-8}$ : while the algorithm with standard population size is faster for several lower dimensions ( $f_{2}$ in $2 \mathrm{D}$ and $3 \mathrm{D}, f_{10}$ in $2 \mathrm{D}, 3 \mathrm{D}$, and $10 \mathrm{D}, f_{13}$ in $2 \mathrm{D}, 3 \mathrm{D}$, and $5 \mathrm{D}, f_{17}$ in $3 \mathrm{D}$ and $10 \mathrm{D}, f_{18}$ in $3 \mathrm{D}$ and $\left.20 \mathrm{D}\right)$ as well as on $f_{6}$ in $20 \mathrm{D}$ and $40 \mathrm{D}$ and for $f_{14}$ in all dimensions but 20, the algorithm with reduced initial population size is sometimes faster for larger dimensions ( $f_{5}$ in 20D and $40 \mathrm{D}$, $f_{8}$ and $f_{12}$ in $\left.40 \mathrm{D}\right)$. Furthermore, one can observe that, in 20D, unsuccessful runs occur for eight of the 24 functions and the functions $f_{3}, f_{4}$, and $f_{19}-f_{24}$ cannot be solved by both algorithms in any of the 15 runs. When compared to the best algorithm of the BBOB'2009 exercise, both algorithms significanly improve the performance on $f_{10}$ (faster by a factor of 1.4), $f_{14}$ (factor of $\geq 1.5$ ), and $f_{11}$ and $f_{15}$ (factor of $>2$, all results in 40D) which is mainly due to the active covariance matrix adaptation [8].

\section{CONCLUSIONS}

When investigating the impact of the initial population size in the IPOP-CMA-ES with active covariance matrix adaptation and mirrored mutation, no general recommendation towards one of the two algorithms $\mathrm{CMA}_{\text {ma }}$ and $\mathrm{CMA}_{\text {mah }}$ can be made. While a lower population size is generally helpful on the sphere function and less effective on the discus function, the positive effect of the lower population size is often more pronounced for larger dimensions with the exception of the attractive sector function where the opposite is the case. As a general conclusion, we remark that the change of the initial population size has overall comparatively small effects.

\section{REFERENCES}

[1] A. Auger, D. Brockhoff, and N. Hansen. Mirrored Sampling in Evolution Strategies With Weighted Recombination. In Genetic and Evolutionary Computation Conference (GECCO 2011), pages 861-868. ACM, 2011.

[2] A. Auger and N. Hansen. A Restart CMA Evolution Strategy With Increasing Population Size. In Congress on Evolutionary Computation (CEC 2005), volume 2, pages 1769-1776. IEEE Press, 2005.

[3] D. Brockhoff, A. Auger, and N. Hansen. On the Effect of Mirroring in the IPOP Active CMA-ES on the Noiseless BBOB Testbed. In GECCO (Companion) workshop on Black-Box Optimization Benchmarking (BBOB'2012). ACM, 2012.

[4] D. Brockhoff, A. Auger, N. Hansen, D. V. Arnold, and T. Hohm. Mirrored Sampling and Sequential Selection for Evolution Strategies. In Conference on Parallel Problem Solving from Nature (PPSN XI), pages 11-21. Springer, 2010.

[5] S. Finck, N. Hansen, R. Ros, and A. Auger. Real-parameter black-box optimization benchmarking 2009: Presentation of the noiseless functions. Technical Report 2009/20, Research Center PPE, 2009. Updated February 2010.

[6] N. Hansen, A. Auger, S. Finck, and R. Ros. Real-parameter black-box optimization benchmarking 2012: Experimental setup. Technical report, INRIA, 2012.

[7] N. Hansen, S. Finck, R. Ros, and A. Auger. Real-parameter black-box optimization benchmarking 2009: Noiseless functions definitions. Technical Report RR-6829, INRIA, 2009. Updated February 2010.

[8] N. Hansen and R. Ros. Benchmarking a weighted negative covariance matrix update on the BBOB-2010 noiseless testbed. In Genetic and Evolutionary Computation Conference (GECCO 2010), pages 1673-1680, New York, NY, USA, 2010. ACM.

[9] G. Jastrebski and D. Arnold. Improving evolution strategies through active covariance matrix adaptation. In IEEE Congress on Evolutionary Computation (CEC 2006), pages 2814-2821, 2006.

[10] K. Price. Differential evolution vs. the functions of the second. In Proceedings of the IEEE International Congress on Evolutionary Computation (ICEO), pages 153-157, 1997. 

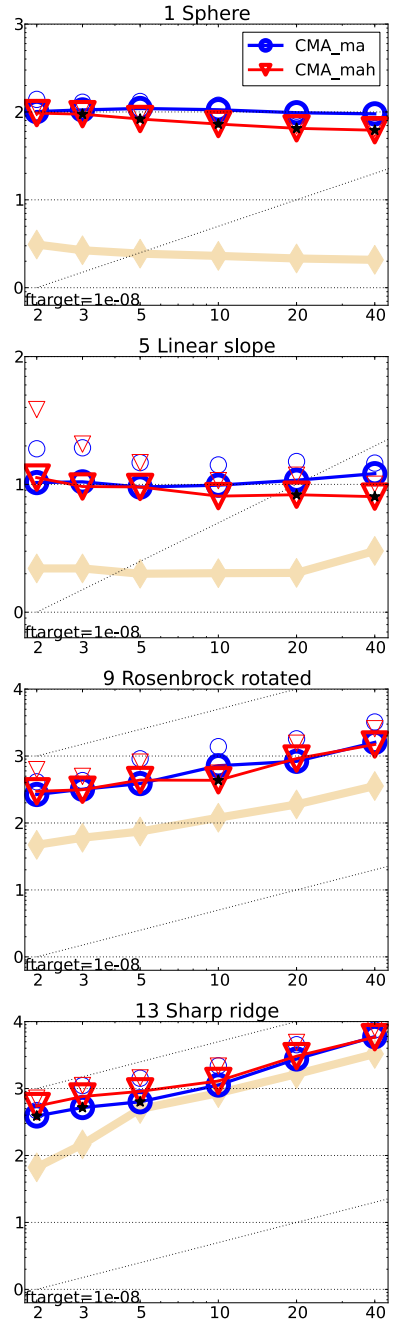

17 Schaffer F7, condition 10
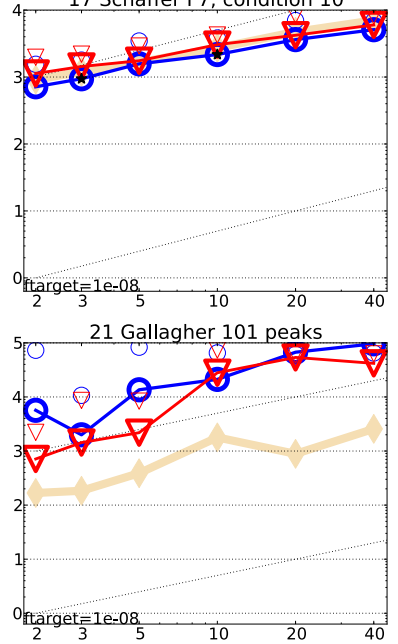
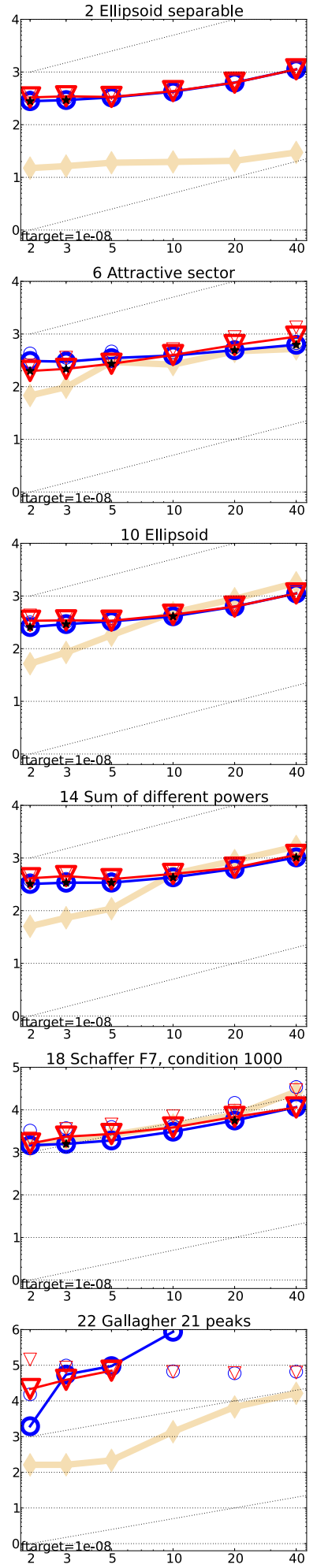
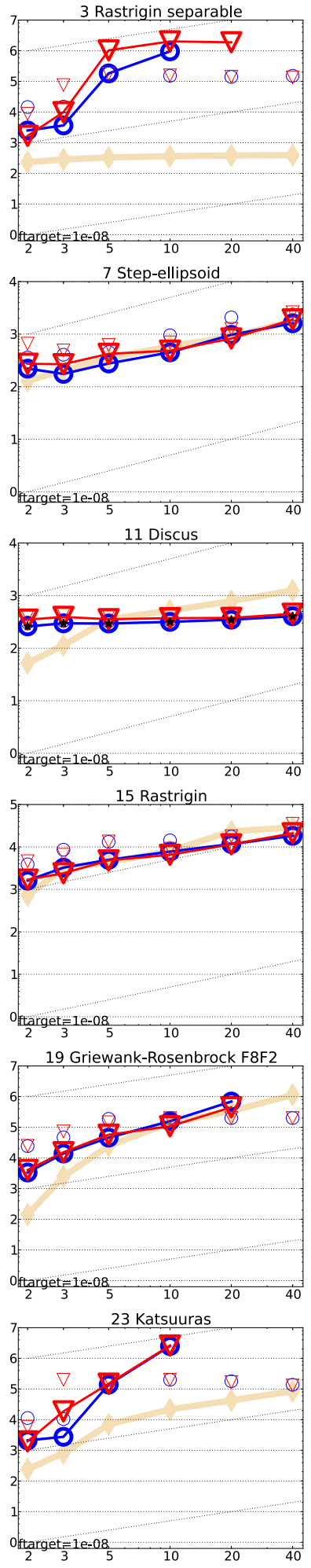
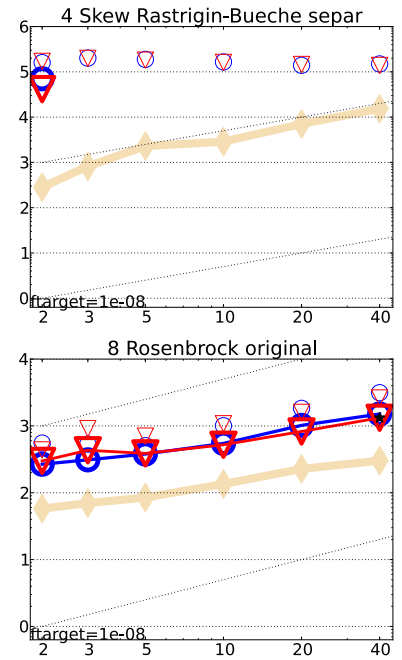

12 Bent cigar
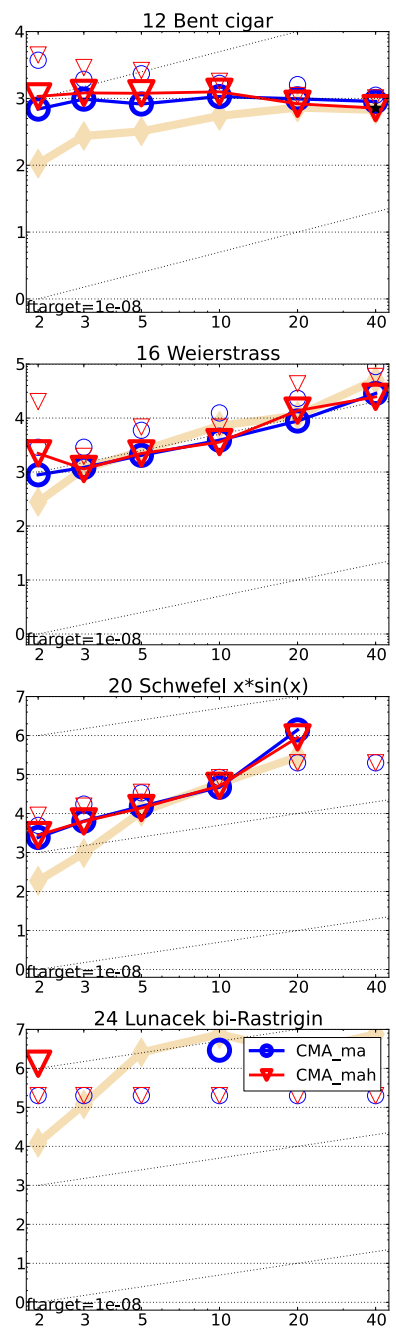

Figure 1: Expected running time (ERT in number of $f$-evaluations) divided by dimension for target function value $10^{-8}$ as $\log _{10}$ values versus dimension. Different symbols correspond to different algorithms given in the legend of $f_{1}$ and $f_{24}$. Light symbols give the maximum number of function evaluations from the longest trial divided by dimension. Horizontal lines give linear scaling, slanted dotted lines give quadratic scaling. Black stars indicate statistically better result compared to all other algorithms with $p<0.01$ and Bonferroni correction number of dimensions (six). Legend: $\circ: \mathrm{CMA}_{\mathrm{ma}}, \nabla: \mathrm{CMA}_{\mathrm{mah}}$. 


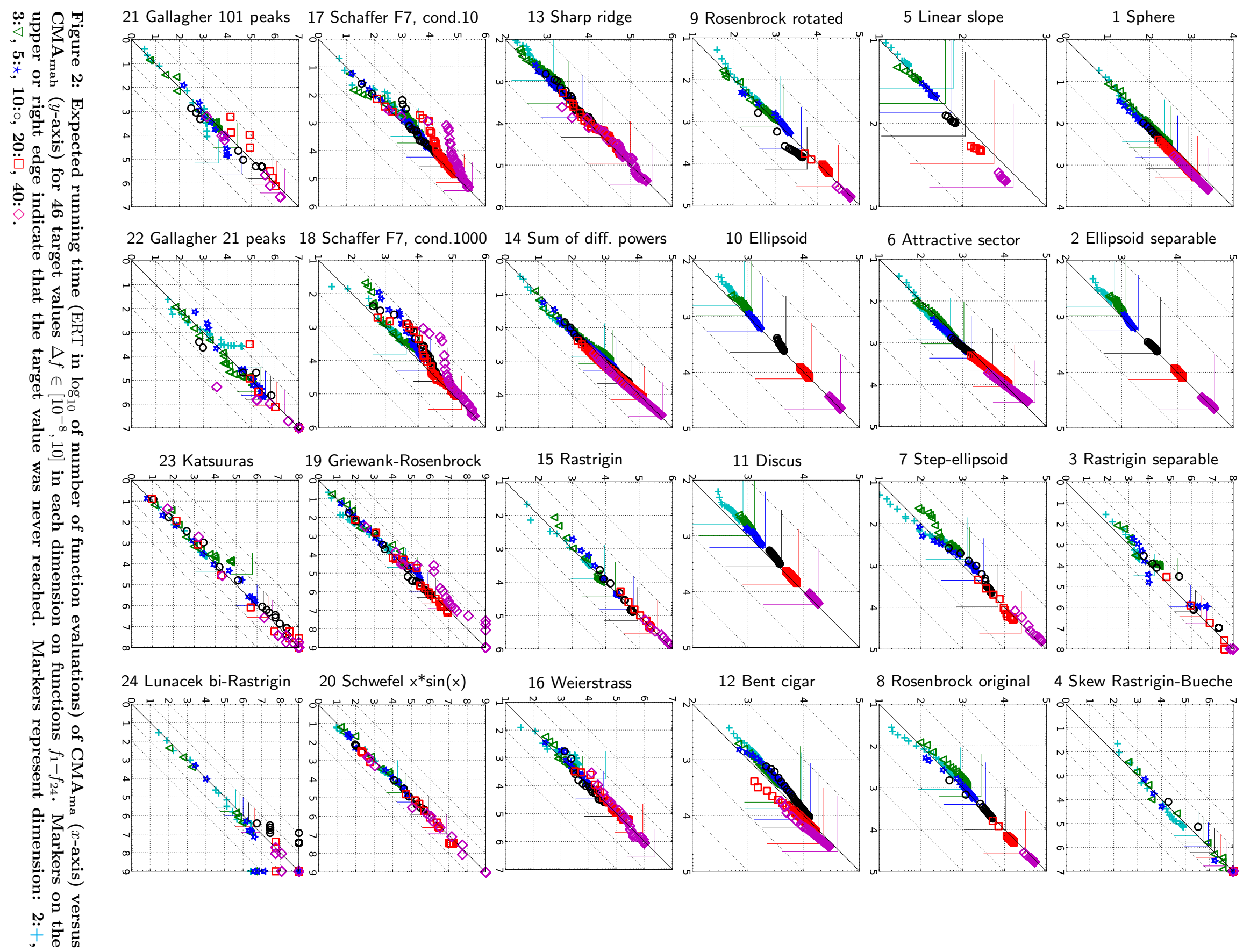



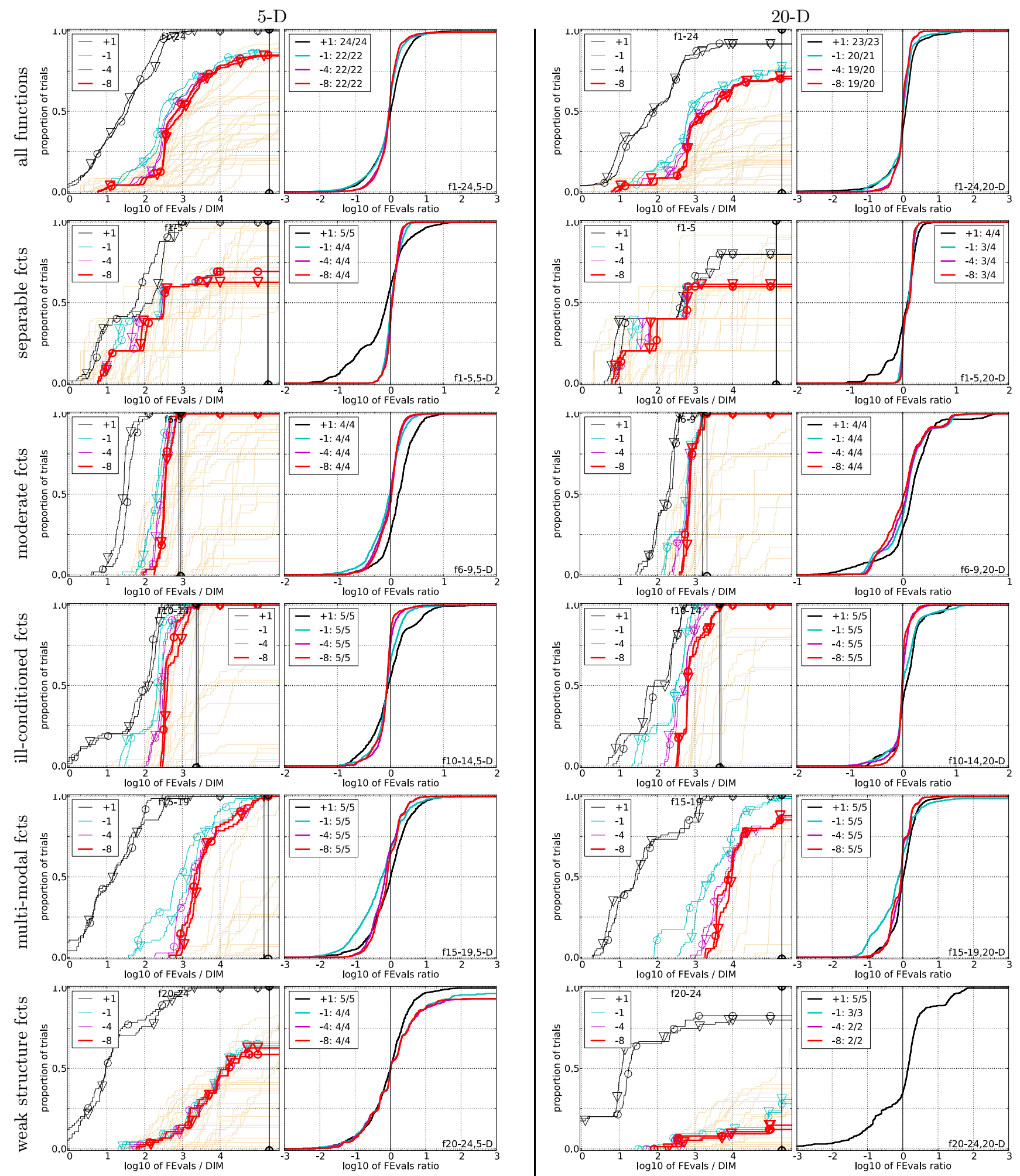

Figure 3: Empirical cumulative distributions (ECDF) of run lengths and speed-up ratios in 5-D (left) and 20D (right). Left sub-columns: ECDF of the number of function evaluations divided by dimension $D$ (FEvals/D) to reach a target value $f_{\text {opt }}+\Delta f$ with $\Delta f=10^{k}$, where $k \in\{1,-1,-4,-8\}$ is given by the first value in the legend, for $\mathrm{CMA}_{\mathrm{ma}}(\circ)$ and CMA mah $(\nabla)$. Light beige lines show the ECDF of FEvals for target value $\Delta f=10^{-8}$ of all algorithms benchmarked during BBOB-2009. Right sub-columns: ECDF of FEval ratios of CMAma divided by $\mathrm{CMA}_{\text {mah }}$, all trial pairs for each function. Pairs where both trials failed are disregarded, pairs where one trial failed are visible in the limits being $>0$ or $<1$. The legends indicate the number of functions that were solved in at least one trial (CMAma first). 
20-D

\begin{tabular}{|c|c|c|c|c|c|c|c|c|c|c|c|c|}
\hline & & & & & & $\Delta f$ & $1 \mathrm{e}+1$ & $1 \mathrm{e}-1$ & $1 \mathrm{e}-3$ & $1 e-5$ & $1 \mathrm{e}-7$ & \#succ \\
\hline$\Delta f$ & $1 \mathrm{e}+1$ & $1 \mathrm{e}-1$ & $1 e-3$ & $1 e-5$ & $1 \mathrm{e}-7$ & \#succ $f_{1}$ & 43 & 43 & 43 & 43 & 43 & $15 / 15$ \\
\hline$f_{1}$ & 11 & 12 & 12 & 12 & 12 & $15 / 15$ 1: CMA & $5.8(0.8)$ & $14(1)$ & $23(1)$ & $32(1)$ & $41(2)$ & $15 / 15$ \\
\hline 1: $\mathrm{CMA}$ & $2.7(3)$ & $11(4)$ & $22(4)$ & $31(5)$ & $40(7)$ & $15 / 15$ 2: $\mathrm{CMA}$ & $3.8(0.8) \star 3$ & $10(1) \star 3$ & $15(1)^{\star 3}$ & $21(1)^{\star 3}$ & $27(1)^{\star 3}$ & $15 / 15$ \\
\hline 2: $\mathrm{CMA}$ & $1.7(1)$ & $8.1(2)$ & $16(2) \star 2$ & $23(2) \star 3$ & $30(3) \star 3$ & $15 / 15 \quad f_{2}$ & 385 & 387 & 390 & 391 & 393 & $15 / 15$ \\
\hline$f_{2}$ & 83 & 88 & 90 & 92 & 94 & $15 / 15$ : $\mathrm{CMA}$ & $22(4)$ & $27(2)$ & $29(2)$ & $31(1)$ & $32(1)$ & $15 / 15$ \\
\hline 1: CMA & $11(3)$ & $14(2)$ & $15(2)$ & $16(2)$ & $17(1)$ & $15 / 152: \mathrm{CMA}$ & $23(5)$ & $28(2)$ & $30(1)$ & $31(1)$ & $32(1)$ & $15 / 15$ \\
\hline 2: CMA & $13(3)$ & $15(2)$ & $16(2)$ & $17(2)$ & $17(2)$ & $15 / 15 f_{3}$ & 5066 & 7635 & 7643 & 7646 & 7651 & $\longdiv { 1 5 / 1 5 }$ \\
\hline$f_{3}$ & 716 & 1637 & 1646 & 1650 & 1654 & $15 / 15$ : $\mathrm{CMA}$ & $7.1(3)$ & $\infty$ & $\infty$ & $\infty$ & $\infty 2.7 e 6$ & $0 / 15$ \\
\hline 1: CMA & $0.74(1)^{\star}$ & $556(737)$ & $554(725)$ & $553(770)$ & $552(728)$ & $7 / 152: \mathrm{CMA}$ & $13(7)$ & $4950(5826)$ & $4945(5457)$ & $4944(5724)$ & $4941(5662)$ & $1 / 15$ \\
\hline 2: CMA & $2.4(2)$ & $3145(3743)$ & $3129(3709)$ & $3121(3761)$ & $3114(3650)$ & $2 / 15 \frac{f_{4}}{2}$ & 4722 & 7666 & 7700 & 7758 & $1.4 \mathrm{e} 5$ & $9 / 15$ \\
\hline \begin{tabular}{l|}
$f_{4}$ \\
\end{tabular} & 809 & 1688 & 1817 & 1886 & 1903 & $\overline{15 / 15} 1: \mathrm{CMA}$ & $\infty$ & $\infty$ & $\infty$ & $\infty$ & $\infty 2.5 e 6$ & $0 / 15$ \\
\hline 1: $\mathrm{CMA}$ & $1.7(2)$ & $\infty$ & $\infty$ & $\infty$ & $\infty 8.8 \mathrm{e} 5$ & $0 / 152: \mathrm{CMA}$ & $\infty$ & $\infty$ & $\infty$ & $\infty$ & $\infty 2.5 e 6$ & $0 / 15$ \\
\hline 2: CMA & $2.5(2)$ & $\infty$ & $\infty$ & $\infty$ & $\infty 8.7 e 5$ & $0 / 15 f_{5}$ & 41 & 41 & 41 & 41 & 41 & $15 / 15$ \\
\hline $\begin{array}{ll}f_{5} \\
\end{array}$ & 10 & 10 & 10 & 10 & 10 & $15 / 151: \mathrm{CMA}$ & $4.6(1)$ & $5.3(1)$ & $5.3(1)$ & $5.3(1)$ & $5.3(1)$ & $15 / 15$ \\
\hline 1: CMA & $3.4(0.8)$ & $4.8(2)$ & $4.8(2)$ & $4.8(2)$ & $4.8(2)$ & $15 / 152: \mathrm{CMA}$ & $3.1(0.6) \star 2$ & $4.1(1)$ & $4.1(1)$ & $4.1(1)$ & $4.1(1)$ & $15 / 15$ \\
\hline 2: $\mathrm{CMA}$ & $3.3(2)$ & $4.7(2)$ & $4.8(2)$ & $4.8(2)$ & $4.8(2)$ & $15 / 15 \frac{f_{6}}{6}$ & 1296 & 3413 & 5220 & 6728 & 8409 & $15 / 15$ \\
\hline$f_{6}$ & 114 & 281 & 580 & 1038 & 1332 & $\overline{15 / 15} 1: \mathrm{CMA}$ & $1.5(0.3)$ & $1.0(0.1)$ & $1.0(0.1)$ & $1.1(0.1)$ & $1.1(0.1)$ & $15 / 15$ \\
\hline 1: CMA & $2.4(1)$ & $2.2(0.9)$ & $1.6(0.4)$ & $1.2(0.2)$ & $1.2(0.2)$ & $15 / 152: \mathrm{CMA}$ & $1.2(0.3)^{\star}$ & $1.0(0.2)$ & $1.1(0.3)$ & $1.2(0.3)$ & $1.3(0.3)$ & $15 / 15$ \\
\hline 2: CMA & $1.7(1.0)$ & $1.7(0.5)$ & $1.3(0.4)$ & $0.94(0.3)$ & $0.92(0.2)^{\star}$ & $15 / 15 f_{7}$ & 1351 & 9503 & 16524 & 16524 & 16969 & $15 / 15$ \\
\hline$f_{7}$ & 24 & 1171 & 1572 & 1572 & 1597 & $15 / 15$ : CMA & $1.6(1)$ & $1.8(0.7)$ & $1.1(0.4)$ & $1.1(0.4)$ & $1.1(0.4)$ & $15 / 15$ \\
\hline 1: CMA & $5.1(3)$ & $0.84(0.6)$ & $0.76(0.5)$ & $0.76(0.5)$ & $0.82(0.5)$ & $15 / 152: \mathrm{CMA}$ & $1.7(1)$ & $1.5(0.6)$ & $0.95(0.3)$ & $0.95(0.3)$ & $0.93(0.3)$ & $15 / 15$ \\
\hline 2: CMA & $3.9(3)$ & $1.4(0.7)$ & $1.2(0.6)$ & $1.2(0.6)$ & $1.2(0.6)$ & $15 / 15 \frac{f_{8}}{8}$ & 2039 & 4040 & 4219 & 4371 & 4484 & $15 / 15$ \\
\hline$f_{8}$ & 73 & 336 & 391 & 410 & 422 & $15 / 15$ : $\mathrm{CMA}$ & $3.1(0.6)$ & $4.4(3)$ & $4.5(3)$ & $4.5(2)$ & $4.5(2)$ & $15 / 15$ \\
\hline 1: CMA & $2.7(1)$ & $3.7(2)$ & $4.0(1)$ & $4.2(1)$ & $4.4(1)$ & $15 / 152: \mathrm{CMA}$ & $2.6(0.5)$ & $3.6(2)$ & $3.7(2)$ & $3.6(2)$ & $3.6(2)$ & $15 / 15$ \\
\hline 2: CMA & $1.8(0.6)$ & $4.0(3)$ & $4.3(2)$ & $4.4(2)$ & $4.5(2)$ & $15 / 15 \frac{f_{9}}{f_{9}}$ & 1716 & 3277 & 3455 & 3594 & 3727 & $\frac{15 / 15}{15 / 15}$ \\
\hline$f_{9}$ & 35 & 214 & 300 & 335 & 369 & $15 / 15$ 1: CMA & $3.4(0.9)$ & $4.3(0.5)$ & $4.4(0.5)$ & $4.4(0.4)$ & $4.4(0.4)$ & $15 / 15$ \\
\hline 1: CMA & $5.9(2)$ & $6.0(2)$ & $5.4(2)$ & $5.3(1)$ & $5.1(1)$ & $15 / 152: \mathrm{CMA}$ & $2.9(0.9)$ & $\begin{array}{l}4.9(3) \\
\end{array}$ & $5.0(3)$ & $\begin{array}{l}4.9(3) \\
\end{array}$ & $\begin{array}{l}4.9(2) \\
\text { la }\end{array}$ & $15 / 15$ \\
\hline 2: $\mathrm{CMA}$ & $4.4(1)$ & $7.5(5)$ & $6.3(3)$ & $6.1(3)$ & $5.8(3)$ & $15 / 15 f_{10}$ & 7413 & 10735 & 14920 & 17073 & 17476 & $15 / 15$ \\
\hline$f_{10}$ & 349 & 574 & 626 & 829 & 880 & $15 / 15$ : $\mathrm{CMA}$ & $1.1(0.2)$ & $0.98(0.1)$ & $0.76(0.0)^{\downarrow 4}$ & $0.70(0.0)^{\downarrow 4}$ & $0.71(0.0)^{\downarrow} 4$ & $15 / 15$ \\
\hline 1: CMA & $2.5(0.8)$ & $2.1(0.3)$ & $2.2(0.2)$ & $1.8(0.2)$ & $1.8(0.2)$ & $15 / 15$ 2: CMA & $1.2(0.2)$ & $1.0(0.1)$ & $0.79(0.1)^{\downarrow 4}$ & $0.71(0.0)^{\downarrow} 4$ & $0.71(0.0) \downarrow 4$ & $15 / 15$ \\
\hline 2: CMA & $2.9(1)$ & $\frac{2.4(0.3)}{763}$ & $2.3(0.3)$ & $1.9(0.2)$ & $1.9(0.2)$ & $15 / 15 \frac{6}{f_{11}}$ & 1002 & 6278 & 9762 & 12285 & 14831 & $15 / 15$ \\
\hline $\begin{array}{c}f_{11} \\
1: \mathrm{CMA}\end{array}$ & $\begin{array}{r}143 \\
5.1(1)\end{array}$ & $\begin{array}{c}763 \\
\mathbf{1 . 3}(0.2) \star 3\end{array}$ & $\begin{array}{c}1177 \\
\mathbf{1 . 0}(0.1) \star 3\end{array}$ & $\begin{array}{c}1467 \\
0.89(0.1) \star 3\end{array}$ & $\begin{array}{c}1673 \\
0.84(0.1) \star 3\end{array}$ & $3 \begin{array}{l}15 / 15 \\
15 / 15\end{array}$ : CMA & $4.3(0.5)^{\star}$ & $0.82(0.1) \star 2$ & $\mathbf{0 . 5 9}(0.0)^{\star 2 \downarrow 4}$ & $0.51(0.0) \star 2 \downarrow 4$ & $0.45(0.0) \star 2 \downarrow$ & $15 / 15$ \\
\hline 2: $\mathrm{CMA}$ & $6.5(2)$ & $1.8(0.3)$ & $\begin{array}{l}1.3(0.2) \\
1.3(0.1)\end{array}$ & $\begin{array}{l}0.09(0.1) \\
1.1(0.1)\end{array}$ & $\begin{array}{l}0.04(0.1) \\
1.0(0.1)\end{array}$ & $15 / 152: \mathrm{CMA}$ & $4.8(0.5)$ & $0.92(0.1)$ & $0.66(0.0) \downarrow 4$ & $0.56(0.0) \downarrow 4$ & $0.49(0.0)^{\downarrow 4}$ & $15 / 15$ \\
\hline \begin{tabular}{l|}
$f_{12}$ \\
\end{tabular} & 108 & 371 & 461 & 1303 & 1494 & $15 / 15 \quad f_{12}$ & 1042 & 2740 & 4140 & 12407 & 13827 & $15 / 15$ \\
\hline 1: $\mathrm{CMA}$ & $6.1(3)$ & $5.4(5)$ & $5.9(4)$ & $2.6(2)$ & $2.6(2)$ & $15 / 15$ 1: CMA & $2.3(2)$ & $3.2(2)$ & $3.1(1)$ & $1.3(0.5)$ & $1.4(0.5)$ & $15 / 15$ \\
\hline 2: $\mathrm{CMA}$ & $6.5(8)$ & $8.1(6)$ & $8.8(5)$ & $3.9(2)$ & $3.8(2)$ & $15 / 152: \mathrm{CMA}$ & $1.2(1)^{\star}$ & $2.6(2)$ & $2.5(1)$ & $1.1(0.4)$ & $1.1(0.3)$ & $15 / 15$ \\
\hline \begin{tabular}{l|}
$f_{13}$ \\
\end{tabular} & 132 & 250 & 1310 & 1752 & 2255 & \begin{tabular}{|l|l}
$15 / 15$ & $f_{13}$
\end{tabular} & 652 & 2751 & 18749 & 24455 & 30201 & $15 / 15$ \\
\hline 1: $\mathrm{CMA}$ & $3.1(2)$ & $4.6(2)$ & $1.2(0.3)$ & $1.3(0.2)$ & $1.2(0.2)$ & $15 / 15$ 1: CMA & $2.9(3)$ & $4.4(2)$ & $0.94(0.4)$ & $1.1(0.5)$ & $1.5(1)$ & $15 / 15$ \\
\hline 2: $\mathrm{CMA}$ & $4.0(4)$ & $5.6(3)$ & $1.7(0.8)$ & $1.7(0.6)$ & $1.6(0.5)$ & $15 / 152: \mathrm{CMA}$ & $3.6(3)$ & $4.2(3)$ & $1.3(0.4)$ & $1.4(0.6)$ & $1.8(0.9)$ & $15 / 15$ \\
\hline$f_{14}$ & 10 & 58 & 139 & 251 & 476 & $15 / 15 \quad f_{14}$ & 75 & 304 & 932 & 1648 & 15661 & $15 / 15$ \\
\hline 1: $\mathrm{CMA}$ & $1.8(3)$ & $3.3(0.6)$ & $3.7(0.7)$ & $4.0(0.8)$ & $3.0(0.4)^{\star}$ & $15 / 15$ 1: CMA & $3.3(1)$ & $2.8(0.4)$ & $2.9(0.3)$ & $3.7(0.3)$ & $0.65(0.0)^{\downarrow} 4$ & $15 / 15$ \\
\hline 2: $\mathrm{CMA}$ & $1.4(1)$ & $2.4(0.6)^{\star}$ & $3.4(0.9)$ & $4.1(1)$ & $3.5(0.4)$ & $15 / 152: \mathrm{CMA}$ & $2.0(0.6)^{\star 2}$ & $1.9(0.3) \star 3$ & $2.3(0.3) \star 3$ & $3.4(0.4)$ & $0.66(0.0) \downarrow 4$ & $15 / 15$ \\
\hline$f_{15}$ & 511 & 19369 & 20073 & 20769 & 21359 & $14 / 15 \frac{f_{15}}{f_{15}}$ & 30378 & $3.1 \mathrm{e} 5$ & $3.2 \mathrm{e} 5$ & $4.5 \mathrm{e} 5$ & $4.6 \mathrm{e} 5$ & $15 / 15$ \\
\hline 1: CMA & $1.0(0.5)$ & $1.1(0.8)$ & $1.1(0.8)$ & $1.1(0.8)$ & $1.1(0.8)$ & $15 / 15$ 1: CMA & $0.62(0.2)^{\downarrow 2}$ & $0.65(0.3)$ & $0.67(0.3)$ & $0.49(0.2)^{\downarrow 3}$ & $0.50(0.2)^{\downarrow 2}$ & $15 / 15$ \\
\hline 2: CMA & $2.0(2)$ & $1.1(0.7)$ & $1.1(0.7)$ & $1.1(0.7)$ & $1.1(0.7)$ & $15 / 15$ 1: 2 : CMA & $\begin{array}{l}0.02(0.2) \\
0.92(0.6)\end{array}$ & $0.64(0.3)$ & $0.65(0.3)$ & $0.48(0.2)^{\downarrow} 2$ & $0.49(0.2)^{\downarrow} 2$ & $15 / 15$ \\
\hline $\begin{array}{c}f_{16} \\
1: \stackrel{C}{\mathrm{CMA}}\end{array}$ & $\begin{array}{r}120 \\
23(2)\end{array}$ & $\begin{array}{l}2662 \\
17(0.8)\end{array}$ & $\begin{array}{l}10449 \\
088(0.6)\end{array}$ & $\begin{array}{c}11644 \\
0.83(0.5)\end{array}$ & $\begin{array}{l}12095 \\
0.83(0.5)\end{array}$ & & 1384 & 77015 & $1.9 \mathrm{e} 5$ & $2.0 \mathrm{e} 5$ & $2.2 \mathrm{e} 5$ & $15 / 15$ \\
\hline $\begin{array}{l}\text { 1: CMA } \\
\text { 2: CMA }\end{array}$ & $\begin{array}{l}2.3(2) \\
2.3(1)\end{array}$ & $\begin{array}{l}1.7(0.8) \\
2.4(2)\end{array}$ & $\begin{array}{l}0.88(0.6) \\
0.94(0.7)\end{array}$ & $\begin{array}{l}0.83(0.5) \\
0.90(0.6)\end{array}$ & $\begin{array}{l}0.83(0.5) \\
0.90(0.6)\end{array}$ & $\mid \begin{array}{l}15 / 15 \\
15 / 15\end{array}$ 1: CMA & $2.2(3)$ & $0.84(0.6)$ & $0.76(0.6)$ & $0.85(0.9)$ & $0.79(0.9)$ & $15 / 15$ \\
\hline$f_{17}$ & 5.2 & 899 & 3669 & 6351 & 7934 & & $2.3(3)$ & $0.90(0.6)$ & $0.92(0.5)$ & $1.3(1)$ & $1.2(0.9)$ & $15 / 15$ \\
\hline 1: $\mathrm{CMA}$ & $3.3(2)$ & $1.1(1)$ & $0.84(0.9)$ & $0.82(0.5)$ & $0.96(0.4)$ & $15 / 15 \quad f_{17}$ & 63 & 4005 & 30677 & 56288 & 80472 & $15 / 15$ \\
\hline 2: $\mathrm{CMA}$ & $3.1(2)$ & $2.1(2)$ & $1.3(0.8)$ & $\begin{array}{l}1.1(0.5) \\
\text { la }\end{array}$ & $1.0(0.4)$ & $15 / 15$ 1: CMA & $2.2(2)$ & $1.4(2)^{\star}$ & $0.70(0.3)$ & $0.79(0.3)$ & $0.82(0.2)^{\downarrow}$ & $15 / 15$ \\
\hline \begin{tabular}{l|}
$f_{18}$ \\
\end{tabular} & 103 & 3968 & 9280 & 10905 & 12469 & $15 / 152: \mathrm{CMA}$ & $2.0(1)$ & $4.0(2)$ & $0.92(0.4)$ & $0.96(0.4)$ & $0.94(0.3)$ & $15 / 15$ \\
\hline 1: $\mathrm{CMA}$ & $0.89(0.7)$ & $0.63(0.5)$ & $0.69(0.6)$ & $0.67(0$ & $0.71(0.5)$ & $15 / 15 \quad f_{18}$ & 621 & 19561 & 67569 & $1.3 \mathrm{e} 5$ & $1.5 \mathrm{e} 5$ & $15 / 15$ \\
\hline 2: CMA & $6.2(2)$ & $1.5(1)$ & $1.2(0.6)$ & $\begin{array}{l}1.1(0.7) \\
\text { la }\end{array}$ & $1.1(0.6)$ & $15 / 15$ 1: CMA & $0.85(0.2)$ & $0.78(0.7)$ & $0.68(0.2)$ & $0.77(0.4)$ & $0.74(0.3)$ & $15 / 15$ \\
\hline$f_{19}$ & 1 & 242 & $1.2 \mathrm{e} 5$ & $1.2 \mathrm{e} 5$ & $1.2 \mathrm{e} 5$ & $15 / 152:$ CMA & $0.95(0.5)$ & $1.2(0.7)$ & $0.79(0.3)$ & $0.88(0.4)$ & $0.91(0.3)$ & $15 / 15$ \\
\hline 1: CMA & $18(13)$ & $59(255)$ & $1.9(2)$ & $1.9(2)$ & $1.9(2)$ & $15 / 15 f_{19}$ & 1 & $3.4 \mathrm{e} 5$ & $6.2 \mathrm{e} 6$ & $6.7 \mathrm{e} 6$ & $6.7 \mathrm{e} 6$ & $15 / 15$ \\
\hline 2: CMA & $17(12)$ & $518(491)$ & $2.4(2)$ & $2.4(2)$ & $2.3(2)$ & $15 / 15$ 1: CMA & $135(44)$ & $3.2(4)$ & $1.0(1.0)$ & $1.6(2)$ & $2.1(2)$ & $4 / 15$ \\
\hline$f_{20}$ & 16 & 38111 & 54470 & 54861 & 55313 & $14 / 152: \mathrm{CMA}$ & $95(46)$ & $2.5(4)$ & $0.91(0.8)$ & $1.3(1)$ & $1.3(1)$ & $6 / 15$ \\
\hline 1: $\mathrm{CMA}$ & $2.4(2)$ & $1.7(2)$ & $1.3(1)$ & $1.3(1)$ & $\begin{array}{l}1.4(1) \\
\text { 1. }\end{array}$ & $15 / 15 \quad f_{20}$ & 82 & $3.1 \mathrm{e} 6$ & $5.5 \mathrm{e} 6$ & $5.6 \mathrm{e} 6$ & $5.6 \mathrm{e} 6$ & $\overline{14 / 15}$ \\
\hline 2: $\mathrm{CMA}$ & $2.3(2)$ & $1.6(2)$ & $1.2(1)$ & $1.2(1)$ & $1.2(1)$ & $15 / 15$ 1: CMA & $3.9(0.7)$ & $1.1(0.7)$ & $5.0(6)$ & $5.0(5)$ & $4.9(5)$ & $2 / 15$ \\
\hline$f_{21}$ & & 1674 & 1705 & 1729 & 1757 & $14 / 152: \mathrm{CMA}$ & $2.5(0.8) \star 2$ & $0.87(0.4)$ & $2.4(3)$ & $3.2(4)$ & $3.2(3)$ & $3 / 15$ \\
\hline 1: $\mathrm{CMA}$ & $2.0(2)$ & $22(18)$ & $39(109)$ & $39(107)$ & $39(106)$ & $13 / 15 \frac{f_{21}}{2}$ & 561 & 14103 & 14643 & 15567 & 17589 & $15 / 15$ \\
\hline 2: $\mathrm{CMA}$ & $3.6(4)$ & $5.7(10)$ & $5.9(11)$ & $6.0(11)$ & $6.1(11)$ & $15 / 15$ 1: $\mathrm{CMA}$ & $3.0(5)$ & $95(126)$ & $92(121)$ & $86(115)$ & $76(101)$ & $7 / 15$ \\
\hline \begin{tabular}{l|}
$f_{22}$ \\
\end{tabular} & 71 & 938 & 1008 & 1040 & 1068 & $14 / 152: \mathrm{CMA}$ & $25(22)$ & $76(118)$ & $73(116)$ & $69(81)$ & $61(72)$ & $8 / 15$ \\
\hline 1: $\mathrm{CMA}$ & $2.0(0.8)$ & $250(369)$ & $465(576)$ & $452(558)$ & $2(542)$ & $7 / 15 \frac{f_{22}}{72}$ & 467 & 23491 & 24948 & 26847 & $1.3 \mathrm{e} 5$ & $12 / 15$ \\
\hline 2: $\mathrm{CMA}$ & $6.3(14)$ & $289(403)$ & $346(523)$ & $336(510)$ & $329(362)$ & $8 / 15$ 1: CMA & $6.7(12)$ & $\infty$ & $\infty$ & $\infty$ & $\infty 1.1$ e6 & $0 / 15$ \\
\hline \begin{tabular}{l|}
$f_{23}$ \\
\end{tabular} & 3.0 & 14249 & 31654 & 33030 & 34256 & $15 / 152: \mathrm{CMA}$ & $187(29)$ & $\infty$ & $\infty$ & $\infty$ & $\infty 1.1 \mathrm{e} 6$ & $0 / 15$ \\
\hline 1: $\mathrm{CMA}$ & $2.5(2)$ & $37(38)$ & $22(32)$ & $21(31)$ & $20(29)$ & $9 / 15 \frac{f_{23}}{2}$ & 3.2 & 67457 & $4.9 \mathrm{e} 5$ & $8.1 \mathrm{e} 5$ & $8.4 \mathrm{e} 5$ & $15 / 15$ \\
\hline 2: $\mathrm{CMA}$ & $1.9(2)$ & $52(71)$ & $23(32)$ & $22(31)$ & $22(30)$ & 9/15 1: CMA & $2.5(3)$ & $255(296)$ & $\infty$ & $\infty$ & $\infty 2.6 \mathrm{e} 6$ & $0 / 15$ \\
\hline & 1622 & $6.4 \mathrm{e} 6$ & $9.6 \mathrm{e} 6$ & $1.3 \mathrm{e} 7$ & $1.3 \mathrm{e} 7$ & $3 / 152: \mathrm{CMA}$ & $2.9(3)$ & $516(567)$ & $\infty$ & $\infty$ & $\infty 2.4 \mathrm{e} 6$ & $0 / 15$ \\
\hline 1: $\mathrm{CMA}$ & $1.3(1)$ & $\infty$ & $\infty$ & $\infty$ & $\infty 1.0 \mathrm{e} 6$ & $0 / 15 f_{24}$ & $1.3 \mathrm{e} 6$ & $5.2 \mathrm{e} 7$ & $5.2 \mathrm{e} 7$ & $5.2 \mathrm{e} 7$ & $5.2 \mathrm{e} 7$ & $3 / 15$ \\
\hline 2: CMA & $1.6(1)$ & $\infty$ & $\infty$ & $\infty$ & $\infty 1.0 e 6$ & $0 / 15$ 1: CMA & $19(22)$ & $\infty$ & $\infty$ & $\infty$ & $\infty 4.0 \mathrm{e} 6$ & $0 / 15$ \\
\hline & & & & & & & $42(48)$ & $\infty$ & $\infty$ & $\infty$ & $\infty 4.0 \mathrm{e} 6$ & $0 / 15$ \\
\hline
\end{tabular}

Table 1: ERT in number of function evaluations divided by the best ERT measured during BBOB-2009 given in the respective first row with the central $80 \%$ range divided by two in brackets for different $\Delta f$ values. \#succ is the number of trials that reached the final target $f_{\mathrm{opt}}+10^{-8}$. 1:CMA is CMA ma and 2:CMA is

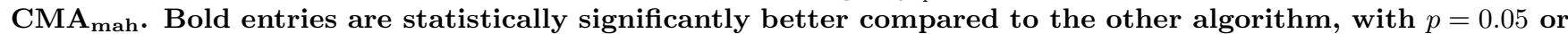
$p=10^{-k}$ where $k \in\{2,3,4, \ldots\}$ is the number following the $\star$ symbol, with Bonferroni correction of 48 . A $\downarrow$ indicates the same tested against the best BBOB-2009. 